\title{
Lebesgue Constant Using Sinc Points
}

\author{
Maha Youssef, ${ }^{1}$ Hany A. El-Sharkawy, ${ }^{1,2}$ and Gerd Baumann ${ }^{1,3}$ \\ ${ }^{1}$ German University in Cairo, New Cairo City 11835, Egypt \\ ${ }^{2}$ Faculty of Science, Ain Shams University, Cairo 11566, Egypt \\ ${ }^{3}$ University of Ulm, Albert-Einstein-Allee 11, 89069 Ulm, Germany \\ Correspondence should be addressed to Maha Youssef; maha.youssef@guc.edu.eg
}

Received 20 April 2016; Revised 20 July 2016; Accepted 18 August 2016

Academic Editor: William J. Layton

Copyright (C) 2016 Maha Youssef et al. This is an open access article distributed under the Creative Commons Attribution License, which permits unrestricted use, distribution, and reproduction in any medium, provided the original work is properly cited.

\begin{abstract}
Lebesgue constant for Lagrange approximation at Sinc points will be examined. We introduce a new barycentric form for Lagrange approximation at Sinc points. Using Thiele's algorithm we show that the Lebesgue constant grows logarithmically as the number of interpolation Sinc points increases. A comparison between the obtained upper bound of Lebesgue constant using Sinc points and other upper bounds for different set of points, like equidistant and Chebyshev points, is introduced.
\end{abstract}

\section{Introduction}

The main point we wish to raise in this article is an effective measure of Lagrange approximation at a set of data defined by a conformal map generating the Sinc points [1].

It is well known that the Lebesgue constant associated with polynomial interpolation always increases at least logarithmically with the number of interpolation points [2]. It is also known that polynomial interpolation at equidistant points has an asymptotic exponential growth rate $[3,4]$. This bad condition combined with Runge's phenomena [5] makes polynomial interpolation at equidistant points sometimes useless for large number of interpolation points. In fact, since Faber and Bernstein $[6,7]$ established their negative results on interpolations, there were many articles demonstrating that with a careful choice of interpolation points an acceptable approximation can be gained if a special set of interpolation points is used [8]. These activities culminated in the famous conjecture by Erdős [9] who proved that there exists an optimal choice of points for Lagrange interpolation. This optimal set of points has so far never been found but some other sets of points might be a near optimal choice [10].

Most of the analytic and numerical estimations of Lebesgue constants discussed in literature are concentrating on Chebyshev points in connection with Lagrange interpolation [8]. There are also a few papers related to cardinal approximations based on Sinc functions [11]. We will compare our results in this paper with the derived formulas for Lagrange approximation using Chebyshev points and with Sinc approximations [11].

To find the upper bound of Lebesgue constant there are two approaches: the analytic approach and the numeric approach. The analytic techniques deliver a true upper bound of the Lebesgue constant using certain type of interpolation points $[2,8,9,11]$. The numeric approach uses a table of Lebesgue constant values to find a least square fitting for these data. It is well known that a least square approximation of such data may fail to represent the asymptotic behavior. In our approach, we use the numeric values of the Lebesgue constant to reach the asymptotic behavior at infinity. For this reason, we use Sinc points approaching infinity as interpolation points in a barycentric form of Lagrange approximation in connection with Thiele's algorithm.

This paper is organized as follows: Section 2 recalls the definition of Lagrange approximation at Sinc points and Lebesgue function/constant. Section 3 presents the barycentric formulas of Lagrange approximation. Section 4 discusses the use of Sinc points in a weighted barycentric formula. Section 5 contains the improved Lebesgue constant using Thiele's algorithm. Section 6 collects some numeric experiments supporting our theoretical findings of the previous sections. Finally, the conclusions are given in Section 7. 


\section{Definition of the Problem}

Assume we have $n+1=2 N+1$ Sinc points on the interval $[-1,1]$. These Sinc points are defined as follows:

$$
x_{k}=\frac{e^{k h}-1}{e^{k h}+1}, \quad k=-N, \ldots, N,
$$

where $h$ is a positive parameter denoting the step length on $\mathscr{R}$. These points are nonequidistant points created by a conformal mapping $\phi(x)=\log ((x+1) /(1-x))$. The function $\phi(x)$ maps the interval $[-1,1]$ to the real line $\mathbb{R}$ for which $x_{k}=\phi^{-1}(k h), k=-N, \ldots, N$. Lagrange approximation at the Sinc points is defined as [1]

$$
p_{n}(x)=\sum_{k=-N}^{N} b_{k}(x) f_{k}
$$

where $f_{k}$ are the function values at Sinc points and $b_{k}(x)$ are the basis functions defined as

$$
b_{k}(x)=\frac{g(x)}{\left(x-x_{k}\right) g^{\prime}\left(x_{k}\right)},
$$

where the function $g(x)$ is given by

$$
g(x)=\prod_{j=-N}^{N}\left(x-x_{k}\right)
$$

There are two properties to influence the performance of the approximation defined in (2), the conditioning and accuracy. The standard measures of these two factors are combined in the Lebesgue constant and Lebesgue function; for recent discussion see $[15,16]$.

The Lebesgue function $\lambda_{n}(x)$ associated with $p_{n}(x)$ defined in (2) is given as

$$
\lambda_{n}(x)=\sum_{j=-N}^{N}\left|b_{j}(x)\right|
$$

The maximum of this function is called Lebesgue constant [17]:

$$
\Lambda_{n}=\max _{x \in[a, b]} \lambda_{n}(x)
$$

In this paper, we derive the asymptotic expression of Lebesgue constant as

$$
\Lambda_{n} \approx c_{1} \log (n)+c_{2}
$$

in which $c_{1}=1 / \pi$ and $c_{2}=1.07618$.

\section{Barycentric Formula}

The basis function $b_{k}(x)$ defined in (3) can be written as [18]

$$
b_{k}(x)=\frac{g(x) w_{k}}{x-x_{k}}
$$

where $g(x)$ is defined as in (4) and the sequence of weights $w_{k}$ is defined as

$$
w_{k}=\frac{1}{g^{\prime}\left(x_{k}\right)}=\frac{1}{\prod_{j=-N, j \neq k}^{N}\left(x_{k}-x_{j}\right)} .
$$

This means that the Lagrange polynomial in (2) can be written as

$$
p_{n}(x)=g(x) \sum_{k=-N}^{N} \frac{w_{k}}{x-x_{k}} f_{k} .
$$

Interpolating the constant function $f=1$ to have

$$
1=g(x) \sum_{k=-N}^{N} \frac{w_{k}}{x-x_{k}}
$$

and dividing (10) by (11), it follows that

$$
\begin{aligned}
p_{n}(x) & =\frac{g(x) \sum_{k=-N}^{N}\left(w_{k} /\left(x-x_{k}\right)\right) f_{k}}{g(x) \sum_{k=-N}^{N}\left(w_{k} /\left(x-x_{k}\right)\right)} \\
& =\frac{\sum_{k=-N}^{N}\left(w_{k} /\left(x-x_{k}\right)\right) f_{k}}{\sum_{k=-N}^{N}\left(w_{k} /\left(x-x_{k}\right)\right)} .
\end{aligned}
$$

Rutishauser called (12) the second barycentric formula. This formula reduces the standard $O\left(n^{2}\right)$ operations in Lagrange interpolation to only $O(n)$ operations. It is also known that (12) is stable in the neighborhood of the interpolation points, which by some authors is called a wellconditioned approximation. In (12), the weights $w_{k}$ appear in the denominator exactly as in the numerator, except without the data factor $f_{k}$. This means that any common factor in all weights $w_{k}$ may be canceled without affecting the value of $p_{n}$.

From (2) and (12) we can derive that

$$
\begin{aligned}
p_{n}(x) & =\frac{g(x) \sum_{k=-N}^{N}\left(w_{k} /\left(x-x_{k}\right)\right) f_{k}}{g(x) \sum_{k=-N}^{N}\left(w_{k} /\left(x-x_{k}\right)\right)} \\
& =\frac{\sum_{k=-N}^{N} b_{k}(x) f_{k}}{\sum_{k=-N}^{N} b_{k}(x)} .
\end{aligned}
$$

This means that the interpolation property $p_{n}\left(x_{k}\right)=f_{k}$ is satisfied independently of the numbers $w_{k}$ as long as they are different from zero. Thus, the function

$$
R_{n}(x)=\frac{g(x) \sum_{k=0}^{n}\left(u_{k} /\left(x-x_{k}\right)\right) f_{k}}{g(x) \sum_{k=0}^{n}\left(u_{k} /\left(x-x_{k}\right)\right)}
$$

interpolates the function $f$ between the given points $x_{k}$ for all choices of the weights $u_{k}$, such that $u_{k} \neq 0, \forall k$ [18]. If $u_{k}=w_{k}$, then $R_{n}$ is the interpolating polynomial; for other choices of $u_{k}, R_{n}$ is a rational function. In [19] it was shown that a rational interpolant represented as a quotient of polynomials is completely determined by its denominator. When the numerator does not have common factors $\left(x-x^{*}\right)$ with the denominator, the weights must alternate their sign 
for the rational function to have no poles in the interval $[-1,1]$ or any another interval of interpolation [18].

Some explicit forms of $u_{k}$ have been derived for some specific types of interpolation points. For details on how to choose $u_{k}$; see $[12,18,20,21]$. For the equidistant points on $[-1,1]$ with spacing $2 / n$, the weights are $u_{k}=(-1)^{k}\left(\begin{array}{l}n \\ k\end{array}\right)[22]$. For Chebyshev points of first kind, $x_{k}=\cos (((2 k+1) /(2 n+$ $2)) \pi$ ), the weights are given by $u_{k}=(-1)^{k}$. Another explicit form of weights can be given for Chebyshev points of second kind, $x_{k}=\cos (k \pi / n)$, by $u_{k}=(-1)^{k} \delta_{k}$, where [12]

$$
\delta_{k}= \begin{cases}\frac{1}{2} & \text { if } k=0 \text { or } k=n \\ 1 & \text { otherwise }\end{cases}
$$

The common feature of all the weights is that they oscillate in sign and have the same magnitude 1, except for the first and last term [23].

The most simple weights, given in [12], are $u_{k}=(-1)^{k}$. In this case (14) is called Berrut's first rational form [12]. For Berrut's first rational form, the following has been proved [12] (Theorem 2.1).

Lemma 1 (see [12]). Let $\left\{x_{k}, f_{k}\right\}, k=0,1, \ldots, n$, be $n+1$ points; then

(1) the rational function

$$
R_{n}(x)=\frac{g(x) \sum_{k=0}^{n}\left((-1)^{k} /\left(x-x_{k}\right)\right) f_{k}}{g(x) \sum_{k=0}^{n}\left((-1)^{k} /\left(x-x_{k}\right)\right)}
$$

interpolates the function $f$ between the points $x_{k}, k=$ $0,1, \ldots, n$, and has no pole on the real line;

(2) $R_{n}(x)=\left(\sum_{k=0}^{n}\left((-1)^{k} /\left(x-x_{k}\right)\right) f_{k}\right) /\left(\sum_{k=0}^{n}\left((-1)^{k} /(x-\right.\right.$ $\left.\left.x_{k}\right)\right)$ ) is a well-conditioned interpolation for a function $f$ between the points $x_{k}, k=0,1, \ldots, n$.

Berrut's first rational approximation has been examined by many authors, mainly to improve the use of equidistant points as interpolation points. The target of all the examinations is studying the Lebesgue constant for such approximation. For example, in [13], the following result for Lebesgue constant $\Lambda_{n}$ has been derived:

$$
\frac{2 n}{4+n \pi} \leq \Lambda_{n} \leq 2+\ln (n) \text {. }
$$

A tighter upper bound of the Lebesgue constant has been obtained in [14]. The improved upper bound satisfies the following inequality:

$$
\begin{aligned}
\Lambda_{n} & \leq 1+\frac{1}{1+\pi^{2} / 24} \ln (n+1) \\
& \approx 1+0.7086 \ln (n+1), \quad \text { for } n \geq 174 .
\end{aligned}
$$

The above results have been extended to more general nodes by Bos et al. in [24]. In their paper, Bos et al. introduced the notion and condition of well-spaced nodes guaranteeing the logarithmic behavior improving Lebesgue constant using
Berrut's first form. They demonstrated that any ordered wellspaced set of points created by a regular function can yield this logarithmic behavior. Note that, using the definition of regularity introduced in [24], we can prove that Sinc points are well-spaced nodes. This means that using Sinc points will guarantee the logarithmic behavior for Lebesgue constant.

In the next section we introduce binomial alternating weights that allow an improvement in the calculations of Lebesgue constant. We later compare the obtained Lebesgue constant for this approximation and the obtained Lebesgue constants mentioned above in (17) and (18) (see Figure 8).

\section{Barycentric Formula at Sinc Points}

4.1. Binomial Weights. Combining Berrut's [12] and Schneider and Werner's [18] result that the weights should be alternating and the concept of regularity offered in [24] will yield a smaller logarithmic level of Lebesgue constant. For this reason we introduce different weights $u_{k}$. We shall show that using these weights in (14) will give a well-conditioned rational approximation with no poles on the real line $\mathbb{R}$. Also we gain a large improvement in the upper bound of Lebesgue constant. To this end, let us rename the Sinc points defined in (1) as

$$
x_{0}<x_{1}<\cdots<x_{n}, \quad \text { here } n=2 N
$$

Now define the weights $u_{k}=(-1)^{k+1}\left(\begin{array}{l}n \\ k\end{array}\right) \neq 0, k=0, \ldots, n$. These weights are alternating and if summed up are zero.

Lemma 2. The polynomial

$$
Q(x)=g(x) \sum_{k=0}^{n} \frac{(-1)^{k+1}\left(\begin{array}{l}
n \\
k
\end{array}\right)}{x-x_{k}}
$$

is different from 0 , for Sinc points $x_{k}$ and $x \in \mathbb{R}$.

Proof. The aim is to show that $Q(x) \neq 0$ for all $x \in \mathbb{R}$. To do so, we let

$$
\sum_{k=0}^{n} \frac{(-1)^{k+1}\left(\begin{array}{l}
n \\
k
\end{array}\right)}{x-x_{k}}=A(x)
$$

We will study the asymptotes and the critical points of $A(x)$ in the interval $\left(x_{k-1}, x_{k}\right) \subset \mathbb{R}$.

From (21) we have

$$
\begin{aligned}
\lim _{x \rightarrow x_{k}} A(x) & =\lim _{x \rightarrow x_{k}} \sum_{k=0}^{n} \frac{(-1)^{k+1}\left(\begin{array}{l}
n \\
k
\end{array}\right)}{x-x_{k}} \\
& = \begin{cases} \pm \infty & \text { if } k \text { is odd } \\
\mp \infty & \text { if } k \text { is even. }\end{cases}
\end{aligned}
$$

This means that $A(x)$ has asymptotes at the Sinc points $x_{k}$. To find the critical points of $A(x)$ in (21), we follow the standard approach by equating the derivative to zero. The critical points in the interval $\left(x_{k-1}, x_{k}\right)$ exist at $x=x_{c}$, where 


$$
\begin{aligned}
x_{c} & =\left(\left(e^{2 h k}-e^{h n}\right)\left(e^{h k}+e^{h+h n / 2}\right)^{2}\left(\begin{array}{c}
n \\
k-1
\end{array}\right)-\left(e^{h k}+e^{h n / 2}\right)^{2}\left(e^{2 h k}-e^{h(2+n)}\right)\left(\begin{array}{l}
n \\
k
\end{array}\right)\right. \\
& -8 e^{2 h(1+n)} \sqrt{\left.e^{-2 h(1-2 k+n)}\left(\begin{array}{c}
n \\
k-1
\end{array}\right)\left(\begin{array}{l}
n \\
k
\end{array}\right)\left(\cosh \left(\frac{h}{2}\right)+\cosh \left(\frac{1}{2} h(1-2 k+n)\right)\right)^{2} \sinh ^{2}\left(\frac{h}{2}\right)\right)} \\
& \cdot\left(\left(e^{h k}+e^{h n / 2}\right)^{2}\left(e^{h k}+e^{h+h n / 2}\right)^{2}\left(\left(\begin{array}{c}
n \\
k-1
\end{array}\right)-\left(\begin{array}{l}
n \\
k
\end{array}\right)\right)\right)^{-1} \cdot
\end{aligned}
$$

This critical point lies approximately at the middle of the interval $\left(x_{k-1}, x_{k}\right)$. The relative local values of $A(x)$ are given

$$
\begin{gathered}
A\left(x_{c}\right)=(-1)^{k} e^{n h}\left[\left(\left(2 e^{2 h}\right)\left(e^{h k}+e^{h n / 2}\right)^{2}\left(e^{h k}+e^{h+h n / 2}\right)^{2}\left(\left(\begin{array}{c}
n \\
k-1
\end{array}\right)-\left(\begin{array}{l}
n \\
k
\end{array}\right)\right)^{2} \sqrt{e^{-2 h(1-2 k+n)}\left(\begin{array}{c}
n \\
k-1
\end{array}\right)\left(\begin{array}{c}
n \\
k
\end{array}\right)\left(\cosh \left(\frac{h}{2}\right)+\cosh \left(\frac{1}{2} h(1-2 k+n)\right)\right)^{2} \sinh ^{2}\left(\frac{h}{2}\right)}\right)\right. \\
\cdot\left(\left[\left(-e^{h k}\left(-1+e^{h}\right)\left(e^{h k}+e^{h n / 2}\right)\left(e^{h k}+e^{h+h n / 2}\right)\left(\begin{array}{l}
n \\
k
\end{array}\right)+e^{2 h+3 h n / 2} \sqrt{\left.e^{h(-4+2 k-3 n)}\left(-1+e^{h}\right)^{2}\left(e^{h k}+e^{h n / 2}\right)^{2}\left(e^{h k}+e^{h+h n / 2}\right)^{2}\left(\begin{array}{c}
n \\
k-1
\end{array}\right)\left(\begin{array}{l}
n \\
k
\end{array}\right)\right)}\right.\right.\right. \\
\left.\left.\left.\cdot\left(e^{h k}\left(-1+e^{h}\right)\left(e^{h k}+e^{h n / 2}\right)\left(e^{h k}+e^{h+h n / 2}\right)\left(\begin{array}{c}
n \\
k-1
\end{array}\right)-4 e^{2 h+3 h n / 2} \sqrt{\left(e^{-2 h(1-2 k+n)}\left(\begin{array}{c}
n \\
k-1
\end{array}\right)\left(\begin{array}{l}
n \\
k
\end{array}\right)\left(\cosh \left(\frac{h}{2}\right)+\cosh \left(\frac{1}{2} h(1-2 k+n)\right)\right)^{2} \sinh ^{2}\left(\frac{h}{2}\right)\right)}\right)\right]\right)^{-1}\right] .
\end{gathered}
$$

This means that $A(x)$ has only one critical point in the interval $x \in\left(x_{k-1}, x_{k}\right)$. Moreover, $A\left(x_{c}\right)$ has the following asymptotic behavior:

$$
A\left(x_{c}\right) \approx(-1)^{k} e^{k h}\left[\kappa(n)+O\left(\frac{1}{k}\right)\right],
$$

where $\kappa(n)$ is a positive function in $n$ growing exponentially as $O\left(e^{n h}\right)$. Since the structure of $\kappa(n)$ is much too complicated to be displayed, we present the plot of this function in Figure 1. Now we end up by the following two cases:

(i) If $x \in\left(x_{k-1}, x_{k}\right)$ and $k$ is even, then $\lim _{x \rightarrow x_{k}^{-}}(-1)^{k+1}\left(\begin{array}{l}n \\ k\end{array}\right) /\left(x-x_{k}\right)=\infty$ and $\lim _{x \rightarrow x_{k-1}^{+}}(-1)^{k}\left(\begin{array}{c}n \\ k-1\end{array}\right) /\left(x-x_{k-1}\right)=\infty$ and $A(x)$ has only one minimal value. To check the sign of this minimal value, we first evaluate $(24)$ at $n=2(N=1)$ to get the minimal value $A\left(x_{c}\right)$ equal to 6.35492 , which is positive number. Also according to (25), for each $n, A\left(x_{c}\right)$ is exponentially increasing in $k$. This means that $A(x)$ is always positive in this case; see Figure 2(a) for demonstration.

(ii) Similarly, if $x \in\left(x_{k-1}, x_{k}\right)$ and $k$ is odd, then $\lim _{x \rightarrow x_{k}^{-}}(-1)^{k+1}\left(\begin{array}{l}n \\ k\end{array}\right) /\left(x-x_{k}\right)=-\infty$ and $\lim _{x \rightarrow x_{k-1}^{+}}(-1)^{k}\left(\begin{array}{c}n \\ k-1\end{array}\right) /\left(x-x_{k-1}\right)=-\infty$ and the sum $A(x)$ has only one maximal value. At $n=2(N=1)$, this maximal value $A\left(x_{c}\right)$ is equal to -6.35492 and has the asymptotic behavior in (25). This means that $A(x)$ is always negative in this case; see Figure 2(a).
This means that, for each $x \in\left(x_{j-1}, x_{j}\right) \subset \mathbb{R}, Q(x) \neq 0$ which completes the proof.

Figure 2(b) presents the Sinc points, as cross " $x$ " with the maximal and minimal value $\left(x_{c}, A\left(x_{c}\right)\right)$, shown as dots. For the calculations we used $n=80$. We note that numerical examinations show that the absolute value of the maximal and minimal value of $A(x)$ increases (in magnitude) exponentially with $n$. An example for $n=80$ is shown in Figure 2.

Inserting the weights $u_{k}=(-1)^{k}\left(\begin{array}{l}n \\ k\end{array}\right)$ into (14) yields the following corollary.

\section{Corollary 3. The approximation}

$$
R_{n}(x)=\frac{g(x) \sum_{k=0}^{n}\left((-1)^{k+1}\left(\begin{array}{l}
n \\
k
\end{array}\right) /\left(x-x_{k}\right)\right) f_{k}}{g(x) \sum_{k=0}^{n}\left((-1)^{k+1}\left(\begin{array}{l}
n \\
k
\end{array}\right) /\left(x-x_{k}\right)\right)}
$$

has no pole on the real line.

Rational approximation defined in (26) is a barycentric formula of polynomial Lagrange approximation defined in (2), where

$$
\begin{gathered}
b_{k}=\frac{g(x) w_{k}}{\left(x-x_{k}\right)} \\
w_{k}=(-1)^{k+1}\left(\begin{array}{l}
n \\
k
\end{array}\right),
\end{gathered}
$$




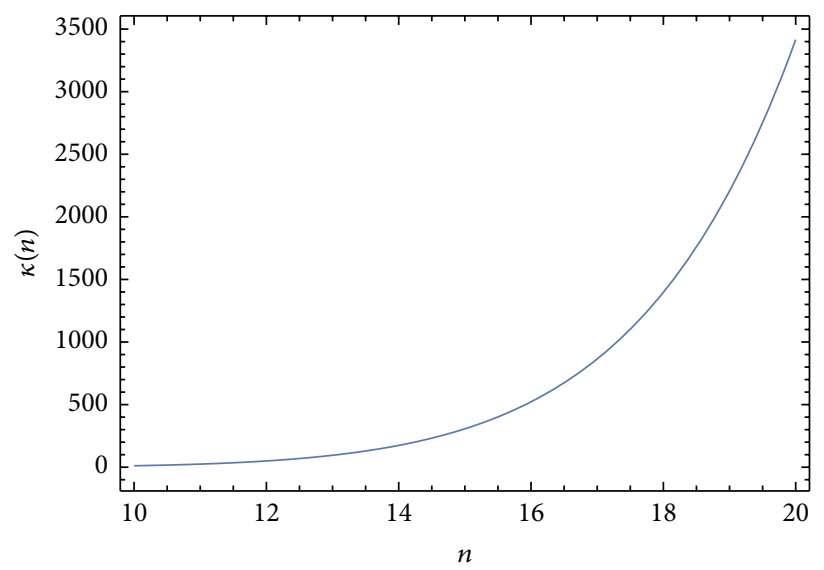

FIGURE 1: The function $\kappa(n)$.

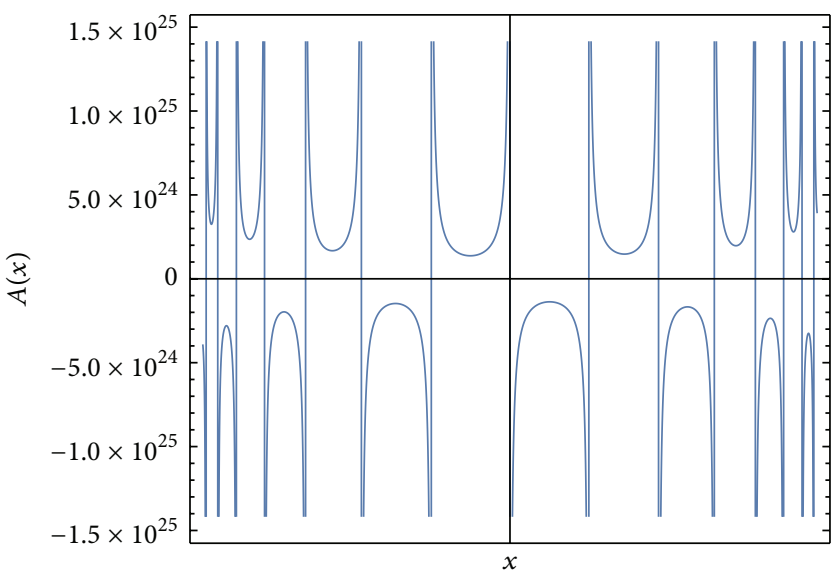

(a)

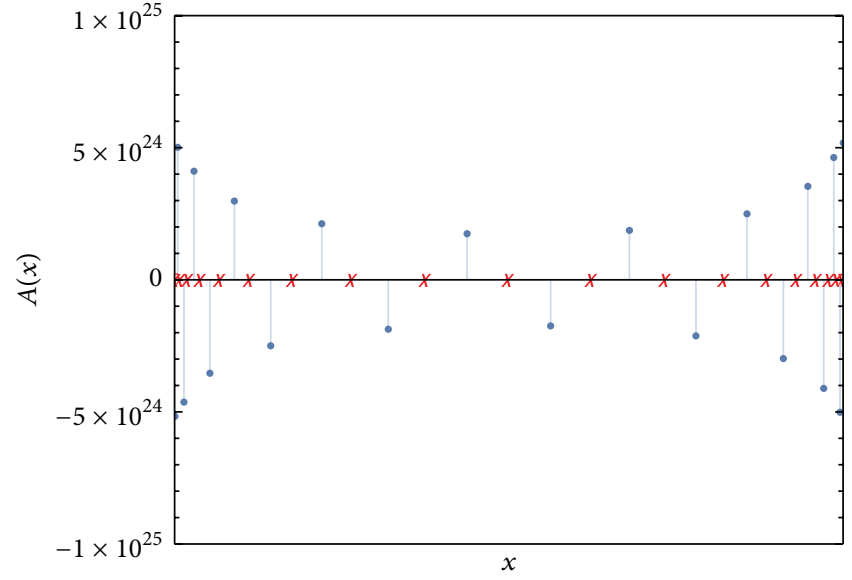

(b)

Figure 2: The sum $A(x)$ in (21) (a). Sinc points with the maximal and minimal values of $A(x)$ using $n=80$ (b).

and $g(x)$ is as defined in (3). As shown in [1], this approximation has an error with exponential decaying rate. Now the approximation takes the form

$$
R_{n}(x)=\frac{\sum_{k=0}^{n}\left((-1)^{k+1}\left(\begin{array}{c}
n \\
k
\end{array}\right) /\left(x-x_{k}\right)\right) f_{k}}{\sum_{k=0}^{n}\left((-1)^{k+1}\left(\begin{array}{l}
n \\
k
\end{array}\right) /\left(x-x_{k}\right)\right)} .
$$

In the calculations of Lebesgue function for (28) at Sinc points, we can observe a high oscillation in the neighborhoods at the end points; see Figure 6. To overcome this problem, there are two solutions. The first solution is to add more (so called fictitious) points taken from the interval $[-1,1]$ to enlarge the computation interval and restrict the evaluation to the interval $[-1,1][25]$. The second solution that we will consider here is to extend the interval $[-1,1]$ by a neighborhood of width $\varepsilon>0$ from the end points and evaluate the approximation only in the interval of practical interest $[-1,1]$ and interval of computations reduced symmetrically by $\varepsilon[26]$.

4.2. Extended Formula. As we will discuss in Numerical Experiments the Lebesgue function for approximation (28) explodes at the end points of the interval; see Figure 6. In order to improve the behavior, one may move the high oscillations out of the interval of practical interest $[-1,1]$. Such an approach was described and effectively used by Klein in [26] and Fornberg in [25]. To do so, we construct data outside the interval of practical interest $[-1,1]$ and introduce an extended formula of the approximation to be defined on the interval of computation $[-1-\varepsilon, 1+\varepsilon]$, where $\varepsilon>0$ is a suitable small positive number. Then the new set of data defined on $[-1-\varepsilon, 1+\varepsilon]$ is interpolated by $(28)$ and examined only in the interval of practical interest $[-1,1][25,26]$. The generation of Sinc points by conformal maps guarantees for finite intervals that the interpolation points are located at the endpoints. Our observation is that most of the Sinc points at the boundaries of the interval are located in a neighborhood with $\varepsilon=0.02$ which is our choice. In such a way, Sinc points are defined as

$$
x_{k}=\frac{(1+\varepsilon) e^{k h}+(-1-\varepsilon)}{e^{k h}+1}, \quad k=-N, \ldots, N .
$$

To show the effectiveness of this extended conformal map, let us discuss Runge's phenomenon [5]. Runge observed that, 


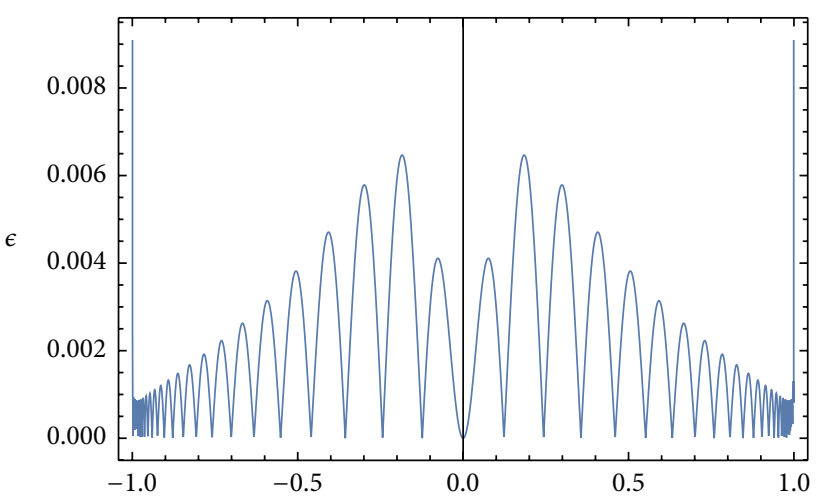

(a)

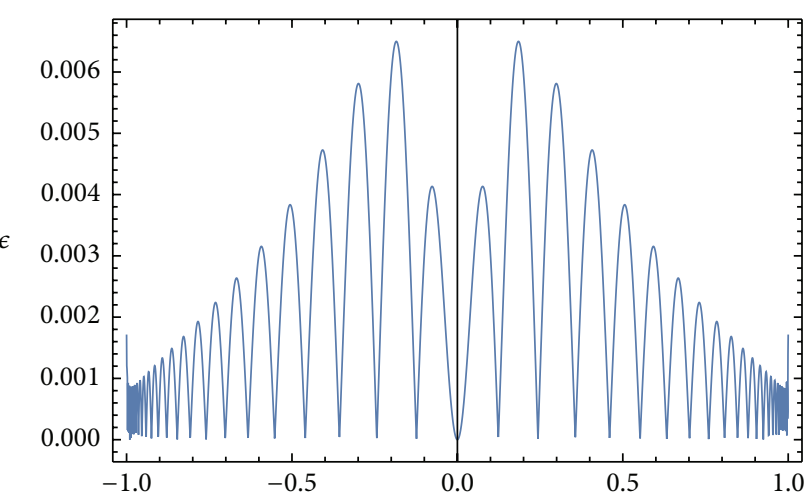

(b)

FIGURE 3: Error in approximating Runge's example on $[-1,1]$ using Sinc points defined in (1) (a). (b) uses Sinc points as defined in (29).

for a test function $f(x)=1 /\left(1+25 x^{2}\right)$ defined on $[-1,1]$, the interpolation and approximation processes deliver bad results at the end points. To overcome this problem, different sets of interpolation points have been introduced $[1,27]$.

To this end, let us use extended Sinc points as interpolation points in (28) and check the behavior of the error. Figure 3 shows two error plots with and without extension of the conformal map in interpolating Runge's function on $[-1,1]$. It is obvious from Figure 3 that the error at the endpoints improves.

\section{Improved Lebesgue Constant}

This section discusses the numerical estimation of the logarithmic behavior of Lebesgue constant $\Lambda_{n}$. We use for the approximation of $\Lambda_{n}$ defined in (28) the Sinc points as given in (29). To examine the behavior of Lebesgue constant as the number of Sinc points approaches infinity, we use Thiele's algorithm.

5.1. Thiele's Algorithm. We shall use Thiele's algorithm for approximating a list of data for Lebesgue constant [28]. Thiele's algorithm works as follows.

Letting $\left\{\left(x_{j}, y_{j}\right)\right\}_{j=0}^{m}$, we define the entries $\Upsilon_{i}^{j}$ as

$$
\begin{aligned}
& \Upsilon_{0}^{j}=y_{j} ; \\
& \Upsilon_{1}^{j}=\frac{x_{j+1}-x_{j}}{\Upsilon_{0}^{j+1}-\Upsilon_{0}^{j}}, \quad j=0,1, \ldots, m-1 ; \\
& \Upsilon_{i}^{j}=\Upsilon_{i-2}^{j+1}+\frac{x_{j+i}-x_{j}}{\Upsilon_{i-1}^{j+1}-\Upsilon_{i-1}^{j}}, \\
& \quad j=0,1, \ldots, m-i ; i=2,3, \ldots, m .
\end{aligned}
$$

Then the rational function $R(x)$ can be defined in a continued fraction form as

$$
R(x)=\Upsilon_{0}^{0}+\frac{x-x_{0}}{\Upsilon_{1}^{0}+} \frac{x-x_{1}}{\Upsilon_{2}^{0}-\Upsilon_{0}^{0}+} \cdots \frac{x-x_{m-1}}{\Upsilon_{m}^{0}-\Upsilon_{m-2}^{0}}
$$

This rational function $R(x)$ interpolates the data $\left\{\left(x_{j}, y_{j}\right)\right\}_{j=0}^{m}$ as $R\left(x_{j}\right)=y_{j}$. Moreover if $m=2 l$, then the rational function $R(x)$ can be written as

$$
R(x)=\frac{p_{l}(x)}{q_{l}(x)}=\frac{\Upsilon_{2 l}^{0} x^{l}+b_{1} x^{l-1}+\cdots+b_{l}}{x^{l}+d_{1} x^{l-1}+\cdots+d_{l}},
$$

where $p_{l}(x)$ and $q_{l}(x)$ are polynomials of degree at most $l$ and $b_{j}$ and $d_{j}$ are some constants and $\Upsilon_{2 l}^{0}$ is the single entry in the last array defined in (30). For the limit as $x \rightarrow \infty$ we have

$$
\lim _{x \rightarrow \infty} R(x)=\Upsilon_{2 l}^{0}
$$

5.2. Estimation of Lebesgue Constant Using Thiele's Algorithm. It is well known that Lebesgue constant of Lagrange interpolation is following the logarithmic inequality $[6,9]$

$$
\Lambda_{n} \leq c_{1} \log (n)+c_{2}
$$

where $c_{1}$ and $c_{2}$ are constants independent of $n$. Different values of the constants $c_{1}$ and $c_{2}$ have been derived for Lagrange approximation and its barycentric formulas [14, 24, 27]. Using Chebyshev points, the optimal choice of $c_{1}$ is $2 / \pi$ and different optimal values of $c_{2}$ have been derived [27] while using the barycentric formula $c_{1}=0.7$ is the optimal and $c_{2}=1[14]$.

To find an estimation for the constants $c_{1}$ and $c_{2}$, we first use least square fitting for a large list of Lebesgue constant data. First let us form a list $\left\{n, \Lambda_{n}\right\}_{n=1}^{600}$. Then we find the best fit for relation (34). Doing so, we found $c_{1} \approx 1 / \pi$ and $c_{2} \approx 1.08$. However, the target is to get the logarithmic behavior of $\Lambda_{n}$ as $n$ approaches infinity. To this end, we define the following function of $n$ :

$$
\rho(n)=\Lambda_{n}-c_{1} \log (n),
$$

where $\Lambda_{n}$ is the calculated Lebesgue constant. The aim is to find the value of $c_{2}$ in an asymptotic estimation:

$$
c_{2}=\lim _{n \rightarrow \infty} \rho(n) \text {. }
$$

Such estimation is delivered by Thiele's algorithm. To get the value of $c_{2}$ we used the value $c_{1}=1 / \pi$ derived from 


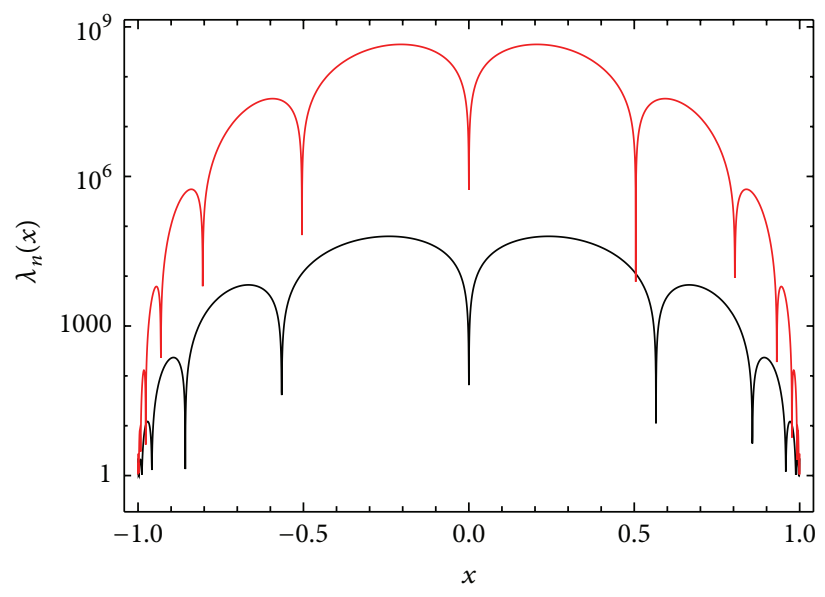

(a)

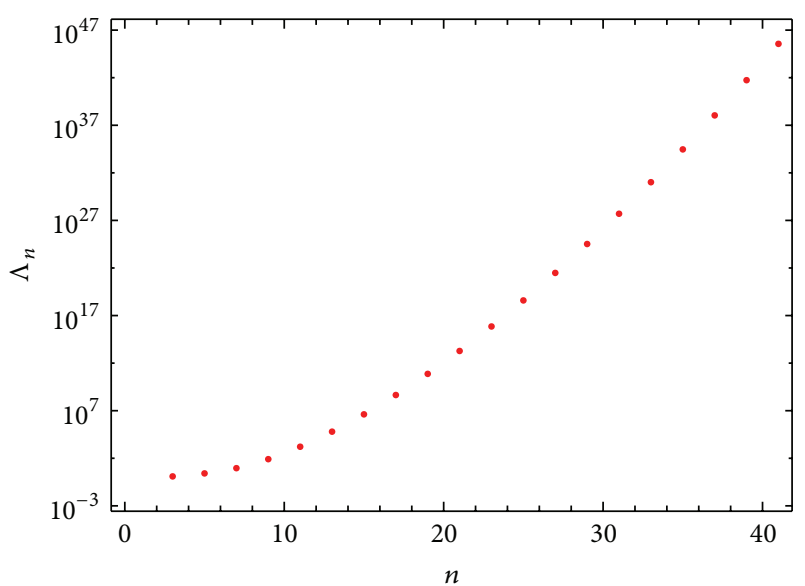

(b)

FIGURE 4: Lebesgue functions for Lagrange approximation (2) at Sinc points (1) with $N=6$ and $N=8$ (a). Lebesgue constant for Lagrange approximation (2) at Sinc points (1) (b).

the least square fitting of the Lebesgue constant data. So the Lebesgue constant is following the logarithmic relation as

$$
\begin{gathered}
\Lambda_{n} \approx \frac{1}{\pi} \log (n)+c_{2}, \\
\rho(n)=\Lambda_{n}-\frac{1}{\pi} \log (n) .
\end{gathered}
$$

First we define the set of data $\{n, \rho(n)\}_{n=1}^{m}$ to be used in Thiele's algorithm (32) to approximate $\rho(n)$ in a rational form. Then $c_{2}=\lim _{n \rightarrow \infty} \rho(n)$ follows from (33). We run the calculations for different numbers of Sinc points equal to $200,400, \ldots, 1000$ to get $c_{2} \approx 1.07618$. Thus we numerically established the following relation for Lebesgue constant:

$$
\Lambda_{n} \approx \frac{1}{\pi} \log (n)+1.07618 .
$$

We note that the constant 1.07618 derived from Thiele's algorithm as $n$ approaches infinity is not far away from the constant 1.08 derived from least square fitting for the Lebesgue constant data.

\section{Numerical Experiments}

The following experiments demonstrate the behavior of Lebesgue constant under different examination scenarios. We start in Example 1 by examining Lagrange's approximation defined in (2) at Sinc points. In Example 2 we examine the use of Sinc points in Berrut's first barycentric formula showing an improvement in the calculation of Lebesgue constant. In Example 3 we examine the evaluation of Lebesgue functions and constant using the binomial barycentric formula discussed in Section 4. Finally we compare all the different computation approaches to figure out the effectiveness of the new weights with Sinc points in barycentric Lagrange approximation $[2,8,11-14]$.

Example 1 (Lagrange at Sinc points). The first experiment is concerned with Lagrange approximation in (2) at Sinc points in (1) for $x \in[-1,1]$ [29]. Here we show that using Sinc points in Lagrange approximation (2) is delivering a large value of the Lebesgue constant. In Figure 4, (a) represents the Lebesgue functions for Lagrange approximation at Sinc points with $N=6$ and $N=8$. The graph shows that the Lebesgue function $\lambda_{n}(x)$ oscillates with a certain amplitude over the interval of interest. The amplitude of the oscillations returns to the same peak level and has a minimal value of 1. In Figure 4, (b) represents the Lebesgue constant $\Lambda_{n}$ as the maximum value of the Lebesgue function $\lambda_{n}(x)$ by varying the number of Sinc points. We definitely observe that Lagrange approximation at Sinc data using the standard approach delivers a large Lebesgue constant that we aim to improve in the following experiments.

Example 2 (Berrut's first formula at Sinc points). The second experiment is concerned with the first Berrut barycentric formula at Sinc points in (1). We aim to improve the Lebesgue constant calculated in Example 1. For this reason we use Sinc points as interpolation points in Berrut's first barycentric formula, that is, with $w_{k}=(-1)^{k}$. In Figure 5, (a) represents the Lebesgue functions for Berrut's first rational approximation at Sinc points with $N=12$ and $N=28$. The maximum of the amplitude over the interval of interest shows a first improvement of the Lebesgue function of the Lagrange approximation examined in the first experiment. In Figure 5, (b) represents Lebesgue constant $\Lambda_{n}$ as the maximum value of the Lebesgue function $\lambda_{n}(x)$ by varying the number of Sinc points. The least square approximation of the calculated Lebesgue constants shows that $\Lambda_{n} \approx 2.0846 \log (n)-1.342$.

Example 3 (binomial weights). Here we discuss Lebesgue function of the barycentric approximation in (28) at Sinc points. The calculations of Lebesgue function and constant are given in Figure 6. Figure 6(a) represents Lebesgue functions for barycentric approximation (28) at Sinc points in (1) with $N=12$ and $N=50$. This graph shows that Lebesgue function $\lambda_{n}(x)$ oscillates with a certain amplitude 


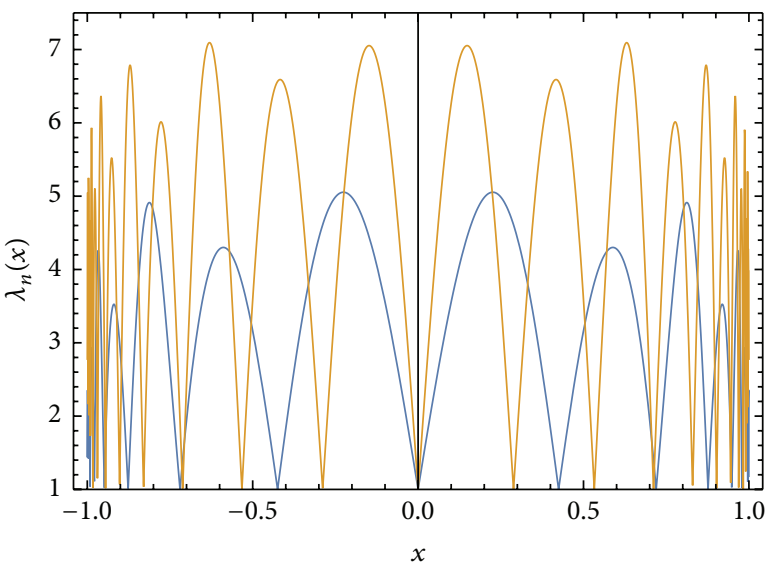

(a)

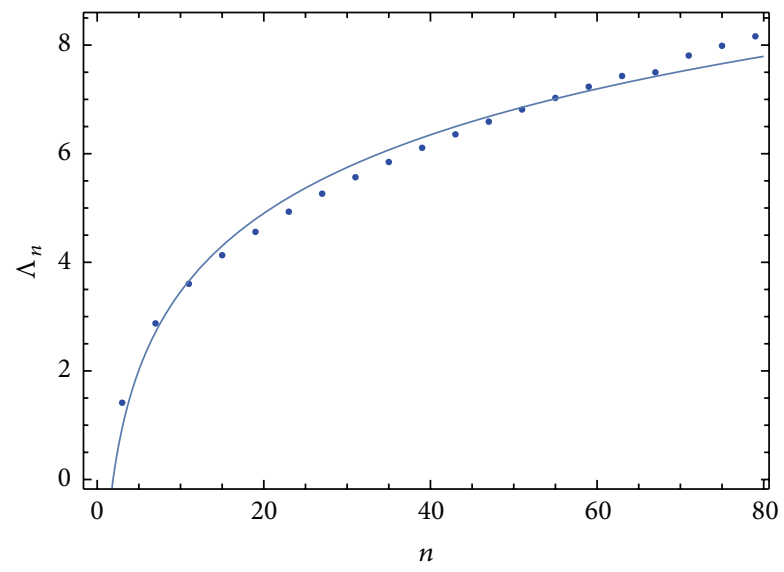

(b)

Figure 5: Lebesgue function for Berrut's first barycentric formula using Sinc points (1). The lower amplitude for $N=12$ and the higher amplitude for $N=28$ (a). Lebesgue constant for first barycentric Berrut form with different Sinc points. The dots represent the calculated Lebesgue constant by varying $N$ from 1 to 40 . The solid line represents the logarithmic least square fitting for these calculations as $2.0846 \log (n)-1.342(\mathrm{~b})$.

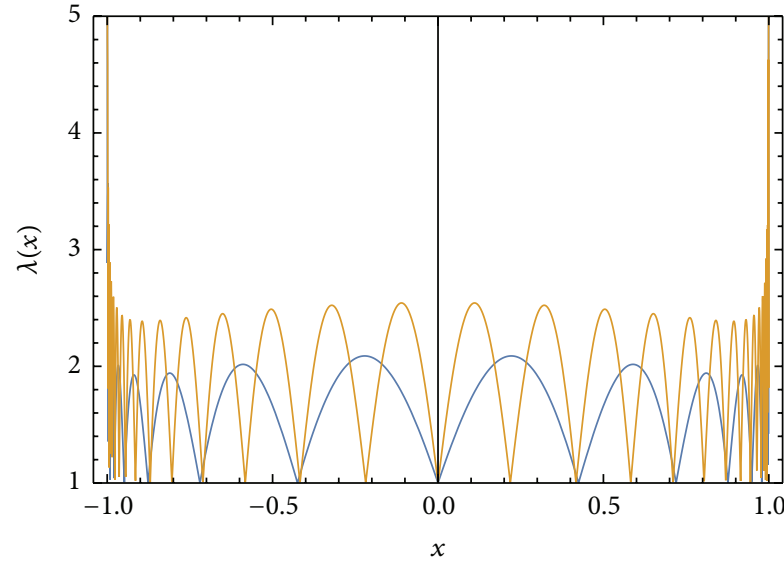

(a)

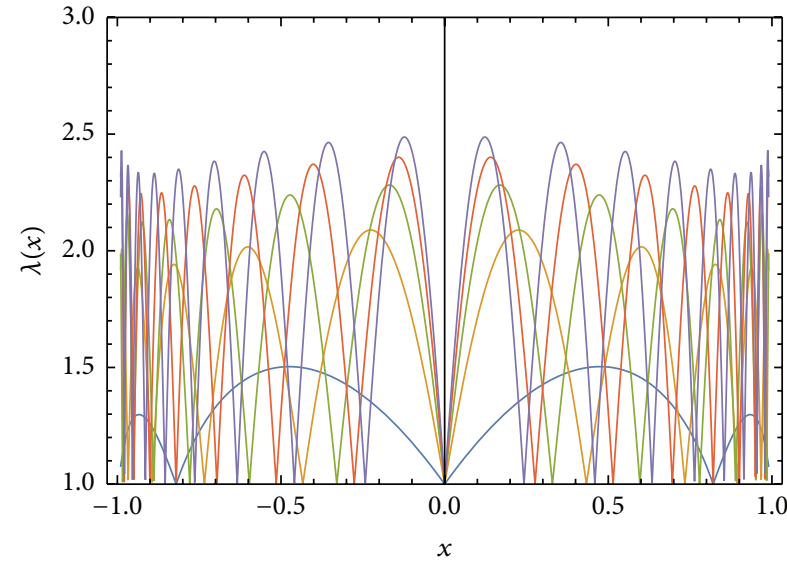

(b)

FIGURE 6: Lebesgue function for barycentric formula (28) using Sinc points (1). The lower amplitude for $N=12$ and the higher amplitude for $N=50$ (a). Lebesgue function for barycentric formula (28) using Sinc points defined in (29). The lowest amplitude for $N=2$ and the highest amplitude for $N=50$ (b).

over the interval of interest except at the neighborhood of the end points of the interval of interest. To avoid these high oscillations at the end points, we use the Sinc points in (29). These calculations of Lebesgue function are given in Figure 6(b). The figure shows definitely the cancellation of the oscillation at the end points. Next, we examine the Lebesgue constant of the barycentric approximation in (28) using Sinc points defined by (29). We first calculate Lebesgue constant for barycentric approximation (28) by varying the number of Sinc points (29) from 2 to 600. These calculations are shown in Figure 7. The dots represent the calculated Lebesgue constants. The solid line represents the logarithmic relation $\Lambda_{n} \approx(1 / \pi) \log (n)+1.07618$. The asymptotic relation is derived by approaching the limit as $n \rightarrow \infty$ for the Thiele approximation of the function $\rho(n)=\Lambda_{n}-(1 / \pi) \log (n)$, where $\Lambda_{n}$ is the Lebesgue constant for (28) using $n$ Sinc points (29).
Example 4 (comparisons). As a final experiment, we compare the Lebesgue constant of binomial barycentric approximation (28) using Sinc points (29) with different results from the literature. We mainly compare our findings with three families of approximations. The first family is Lagrange's approximation at Chebyshev points. In Figure 8 we observe that Lebesgue constant for the binomial barycentric formula at Sinc points is down the upper and lower bounds derived by Rivlin and Brutman [2, 8]. In the second family of approximation, we compare our findings with Sinc approximation. This approximation has an upper bound of Lebesgue constant $\Lambda_{n}=(2 / \pi)(\log (n)+\gamma+\log (2))$ [11]. Figure 8 shows that the derived logarithmic relation of Lebesgue constant for binomial formula (28) at Sinc points is down the upper bound of Lebesgue constant for Sinc approximation derived in [11]. As a final comparison, we compare our findings with Berrut's first formula using weights $(-1)^{k}$ at different types of points. 


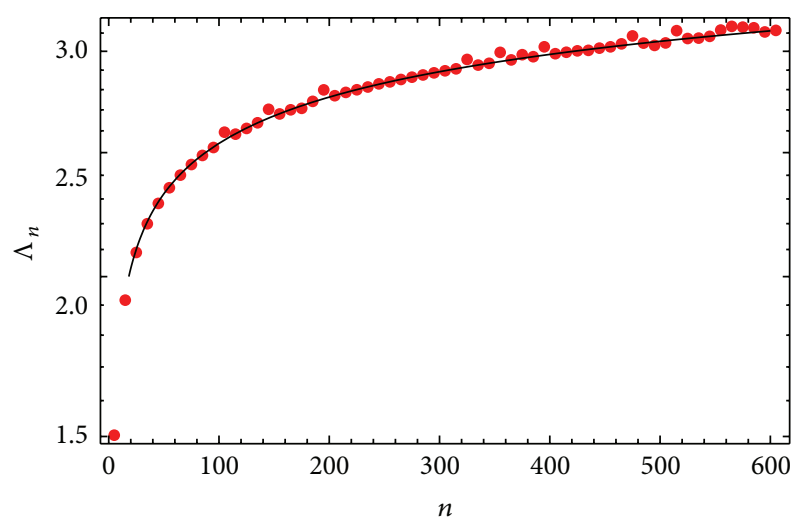

FIGURE 7: Lebesgue constant for (28) with different Sinc points. The dots represent the calculated Lebesgue constant by varying $n$ from 2 to 600 . The solid line represents the logarithmic relation, $\Lambda_{n} \approx$ $(1 / \pi) \log (n)+1.07618$, derived via Thiele's algorithm.

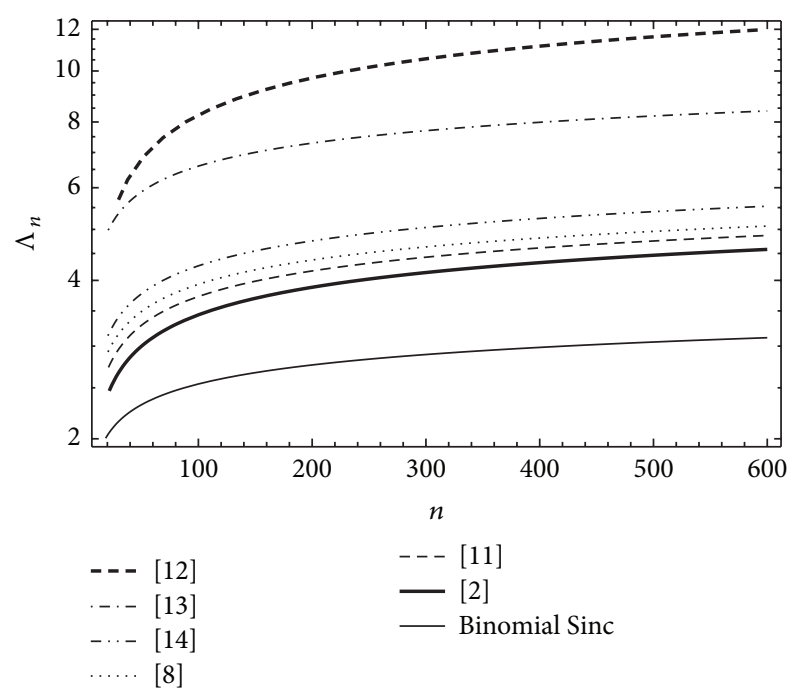

Figure 8: Lebesgue constants for different set of points. Comparison between the logarithmic relation $(1 / \pi) \log (n)+1.07618$ and the obtained results in $[2,8,11-14]$.

We compare our findings with Berrut's formula at Sinc points and at equidistant points. For the Sinc points, we use the calculations of Example 2 and for the equidistant points we used the upper bound derived in [13] and the improved upper bound derived in [14]. Figure 8 shows that using binomial weights will deliver much smaller values for the Lebesgue constant.

\section{Conclusion}

We discussed the Lebesgue constant of Lagrange approximation at Sinc points. We introduced a weighted barycentric form of Lagrange interpolation, which delivers an improvement in Lebesgue constant. Moreover, we used Thiele's algorithm on numeric values of Lebesgue constant to study the behavior as the number of used Sinc points approaches infinity. Finally, besides other advantages from using Sinc points as interpolation points in Lagrange interpolation formula, specially in a finite interval, we can conclude that Lagrange approximation at Sinc points delivers approximation results closer to the conjectured optimal approximation than Chebyshev approximations using barycentric formulas.

\section{Competing Interests}

The authors declare that they have no competing interests.

\section{Acknowledgments}

The authors acknowledge partial financial support by the DAAD/BMBF Project no. 57128284. The authors also acknowledge the valuable discussions with F. Stenger and his comments.

\section{References}

[1] F. Stenger, M. Youssef, and J. Niebsch, "Improved approximation via use of transformations," in Multiscale Signal Analysis and Modeling, X. Shen and A. I. Zayed, Eds., pp. 25-49, Springer, New York, NY, USA, 2013.

[2] L. Brutman, "On the Lebesgue function for polynomial interpolation," SIAM Journal on Numerical Analysis, vol. 15, no. 4, pp. 694-704, 1978.

[3] L. N. Trefethen and J. A. C. Weideman, "Two results on polynomial interpolation in equally spaced points," Journal of Approximation Theory, vol. 65, no. 3, pp. 247-260, 1991.

[4] A. Schönhage, "Fehlerfortpflanzung bei Interpolation," Numerische Mathematik, vol. 3, pp. 62-71, 1961.

[5] C. Runge, "Über empirische Funktionen und die Interpolation zwischen äquidistanten Ordinaten," Zeitschrift für Mathematik und Physik, vol. 46, pp. 224-243, 1901.

[6] G. Faber, "Uber die interpolatorische Darstellung stetiger Funktionen," Jahresbericht der Deutschen Mathematiker-Vereinigung, vol. 23, pp. 192-210, 1914.

[7] S. Bernstein, "Sur l'ordre de la meilleure approximation des fonctions continues par les polynômes de degré donné," Mém. Cl. Sci. Acad. Roy. Belg, vol. 4, pp. 1-103, 1912.

[8] T. Rivlin, "The lebesgue constants for polynomial interpolation," in Functional Analysis and Its Applications, H. Garnir, K. Unni, and J. Williamson, Eds., vol. 399 of Lecture Notes in Mathematics, pp. 422-437, Springer, Berlin, Germany, 1974.

[9] P. Erdös, "Problems and results on the theory of interpolation. II," Acta Mathematica Academiae Scientiarum Hungarica, vol. 12, pp. 235-244, 1961.

[10] C. De Boor and A. Pinkus, "Proof of the conjectures of Bernstein and Erdös concerning the optimal nodes for polynomial interpolation," Journal of Approximation Theory, vol. 24, no. 4, pp. 289-303, 1978.

[11] F. Stenger, H. A. El-Sharkawy, and G. Baumann, "The Lebesgue constant for sinc approximations," in New Perspectives on Approximation and Sampling Theory: Festschrift in Honor of Paul Butzer's 85th Birthday, A. I. Zayed and G. Schmeisser, Eds., Applied and Numerical Harmonic Analysis, chapter 13, pp. 319335, Birkhäauser, Basel Switzerland, 2014.

[12] J.-P. Berrut, "Rational functions for guaranteed and experimentally well-conditioned global interpolation," Computers \& Mathematics with Applications, vol. 15, no. 1, pp. 1-16, 1988. 
[13] L. Bos, S. De Marchi, and K. Hormann, "On the Lebesgue constant of Berrut's rational interpolant at equidistant nodes," Journal of Computational and Applied Mathematics, vol. 236, no. 4, pp. 504-510, 2011.

[14] R.-J. Zhang, "An improved upper bound on the Lebesgue constant of Berrut's rational interpolation operator," Journal of Computational and Applied Mathematics, vol. 255, pp. 652-660, 2014.

[15] E. V. Strelkova and V. T. Shevaldin, "On Lebesgue constants of local parabolic splines," Proceedings of the Steklov Institute of Mathematics, vol. 289, supplement 1, pp. 192-198, 2015.

[16] S. Ghili and G. Iaccarino, "Reusing Chebyshev points for polynomial interpolation," Numerical Algorithms, vol. 70, no. 2, pp. 249-267, 2015.

[17] M. J. D. Powell, Approximation Theory and Methods, Cambridge University Press, Cambridge, UK, 1981.

[18] C. Schneider and W. Werner, "Some new aspects of rational interpolation," Mathematics of Computation, vol. 47, no. 175, pp. 285-299, 1986.

[19] J.-P. Berrut and H. D. Mittelmann, "Matrices for the direct determination of the barycentric weights of rational interpolation," Journal of Computational and Applied Mathematics, vol. 78, no. 2, pp. 355-370, 1997.

[20] M. S. Floater and K. Hormann, "Barycentric rational interpolation with no poles and high rates of approximation," Numerische Mathematik, vol. 107, no. 2, pp. 315-331, 2007.

[21] J. M. Carnicer, "Weighted interpolation for equidistant nodes," Numerical Algorithms, vol. 55, no. 2-3, pp. 223-232, 2010.

[22] H. R. Schwarz, Numerische Mathematik, Teubner, Stuttgart, Germany, 4th edition, 1997, English Translation: Numerical Analysis: A Comprehensive Introduction, John Wiley \& Sons, New York, NY, USA, 2nd edition, 1989.

[23] P. Henrici, Essentials of Numerical Analysis, John Wiley \& Sons, New York, NY, USA, 1982.

[24] L. Bos, S. De Marchi, K. Hormann, and J. Sidon, "Bounding the Lebesgue constant for Berrut's rational interpolant at general nodes," Journal of Approximation Theory, vol. 169, pp. 7-22, 2013.

[25] B. Fornberg, A Practical Guide to Pseudospectral Methods, vol. 1 of Cambridge Monographs on Applied and Computational Mathematics, Cambridge University Press, Cambridge, UK, 1996.

[26] G. Klein, "An extension of the Floater-Hormann family of barycentric rational interpolants," Mathematics of Computation, vol. 82, no. 284, pp. 2273-2292, 2013.

[27] S. J. Smith, "Lebesgue constants in polynomial interpolation," Annales Mathematicae et Informaticae, vol. 33, pp. 109-123, 2006.

[28] F. Stenger, Numerical Methods Based on Sinc and Analytic Functions, Springer, New York, NY, USA, 1993.

[29] F. Stenger, Handbook of Sinc Methods, CRC Press, New York, NY, USA, 2011. 


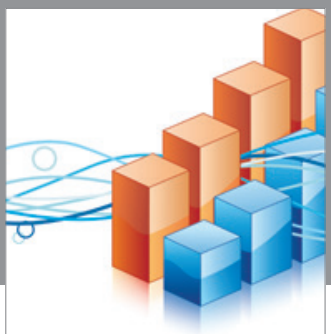

Advances in

Operations Research

vatem alat4

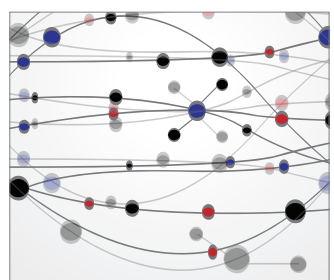

\section{The Scientific} World Journal
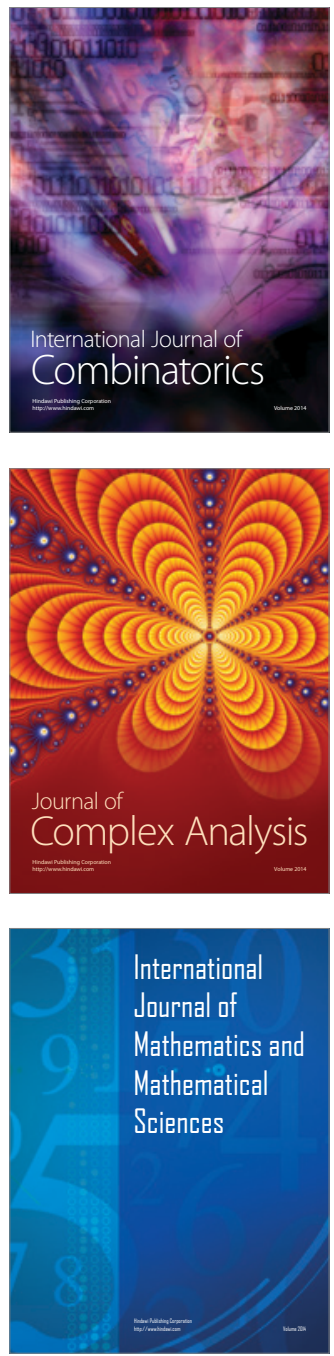
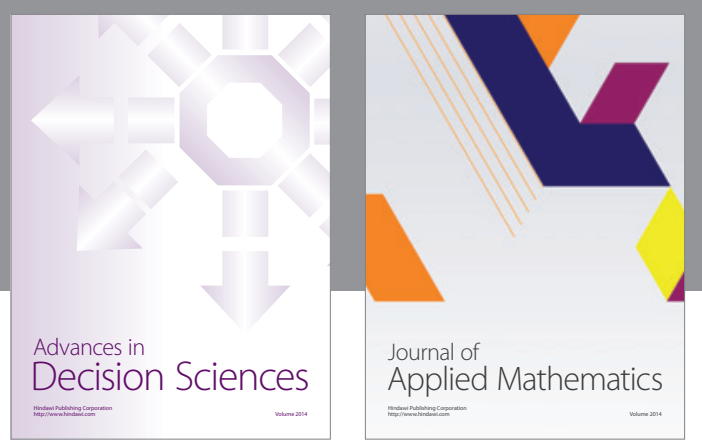

Algebra

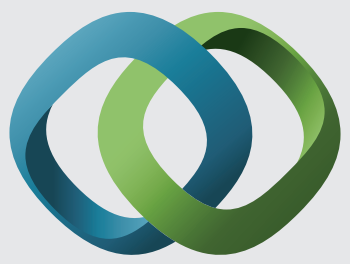

\section{Hindawi}

Submit your manuscripts at

http://www.hindawi.com
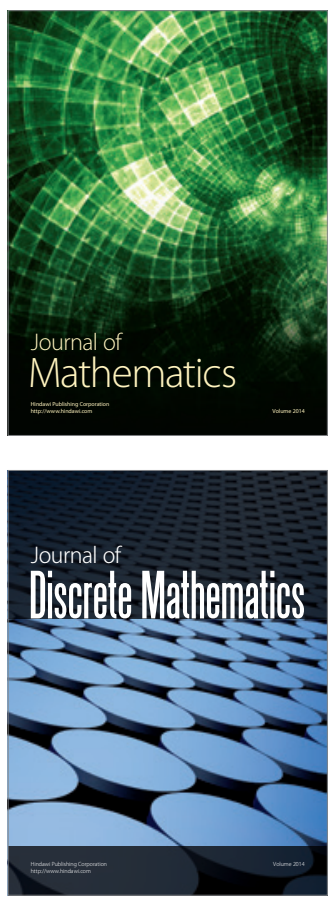

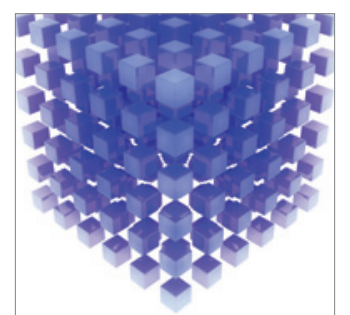

Mathematical Problems in Engineering
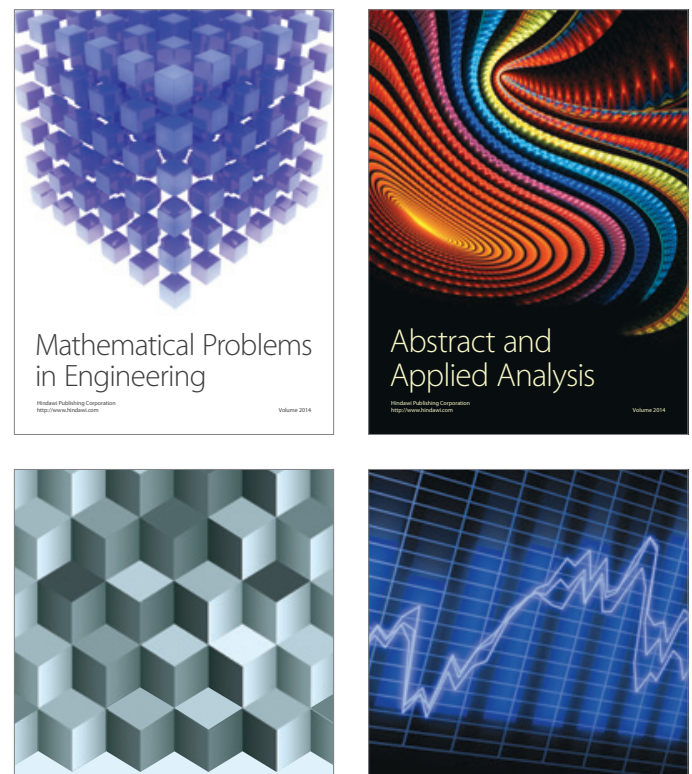

Journal of

Function Spaces

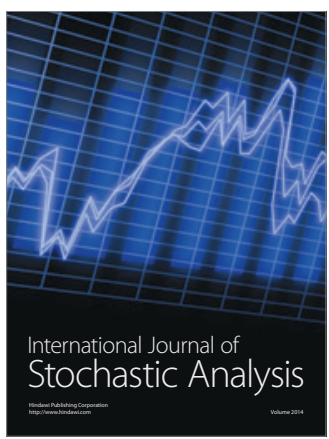

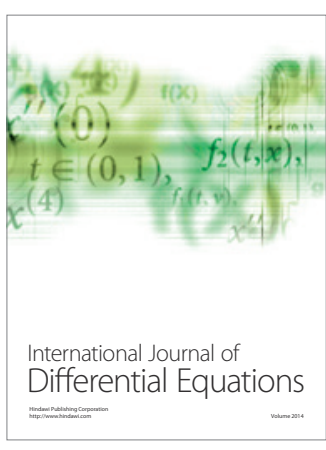
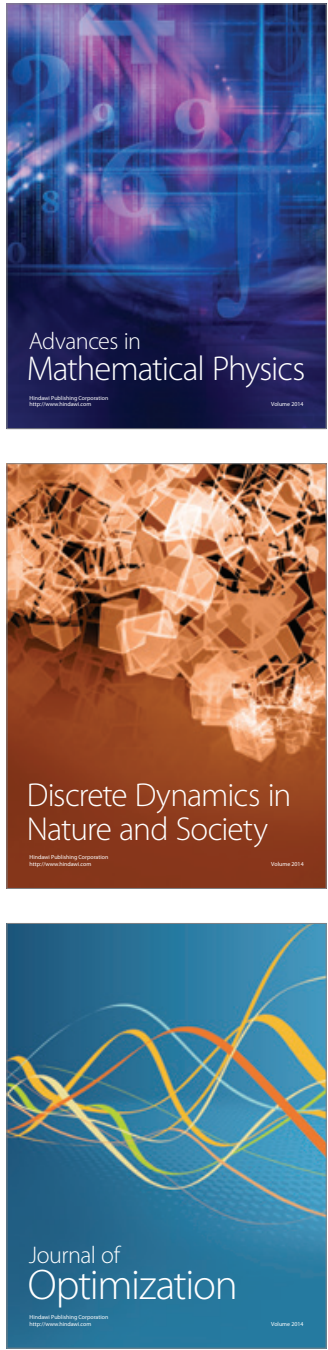